\title{
An Economic analysis of Gynecological Cancer patients in Coimbatore District
}

\author{
Dr.R.Maheshvari* and Mrs. R.Meenakshi** \\ *Assistant Professor in Economics, Department of Humanities, Coimbatore Institute of \\ Technology, Coimbatore -641014 \\ ** Assistant Professor in Economics, Department of Economics, Nirmala College for \\ Women, Coimbatore
}

\begin{abstract}
Economic cost analysis or cost-effectiveness analysis has become apparent in determining the health-care practices. The greatest effect on increase in Gynecological cancer is one of India's greatest public health challenges. Public expenditure on cancer in India remains below US\$10 per person (compared with more than US\$100 per person in high-income countries), and overall public expenditure on health care is still only slightly above $1 \%$ of gross domestic product. Out-of-pocket payments, which account for more than three-quarters of cancer expenditures in India, are one of the greatest threats to patients and families, and a cancer diagnosis is increasingly responsible for catastrophic expenditures that negatively affect not only the patient but also the welfare and education of several generations of their family. (CS Pramesh 2014)
\end{abstract}

A major challenge for the developing countries is to find strategies in which their scarce resources can be properly utilized in controlling this Gynecological Cancer; else this could become a major hindrance to the both Women and socioeconomic development of economically emerging nations. The health of Indian women is intrinsically linked to their status in society. Research on women's health status has found that the contributions of Indian women to families are often overlooked, and instead they are viewed as economic burden too. 
In recent years, market forces and political processes have generated growing interest with regard to the economic costs of diseases to the individual, family, institution, and society. The increasing influence of market forces in day-today practice, lack of proper treatment guidelines among physicians, awareness among patients regarding themselves as consumers, and lack of political willpower among the governing agencies, adds to these cost inflations. Hence, there is a growing need in health sector to live within budgets, more so in a country like India. In time to come, cost analyses will be an important component in policy making, for effective health care delivery (K Sharma 2009)

Keywords: Gynecological cancer, socioeconomic development, Cost Inflation, Budget

\section{Introduction}

Healthcare is defined as scientific and medical understanding of how to treat and prevent disease. Economics can, however, provide useful insights to the design and implementation of the systems to provide healthcare, as well as in the process of prioritizing interventions to make the best use of scarce resources. Mortality rates of cervical cancer in the world are 13.1/105 and 6.9/105, respectively. In India, it is estimated that there are 96,922 new cervical cancer cases and 60,078 deaths and ranked second among women cancers. The incidence and mortality rates are 14.7/105 and 9.2/105 , respectively, although the incidence varies within Indian populationBalasubramaniamet al (2020). The scenario of Healthcare in India presents a rather dismal picture. This is all the more glaring in case of women's health. Although women experience many health problems similar to those of men, there are some problems which are peculiar to them because of the biological factors. Thus, the healthcare needs of women are more complex and varied. However, in a large number of cases these needs are overlooked 
and proper attention is not given to the health of women due to a number of social and cultural factors in the Indian society for a long period of time.

In India, cancer has taken the second place for most of the mortality rates next to cardiovascular diseases. Women's health is largely neglected in general and this led to the lack of medical treatment for the women cancer patients. Existence of gender discrimination in providing medical facilities to the women is clearly apparent in India.India's cancer cases could increase by 12per cent in the next five years, with 1.5 million people projected to suffer from the noncommunicable disease by 2025, up from 1.39 million in 2020, according to data based on current trends from the cancer report by Indian Council of Medical Research (ICMR). The cancer incidence (new cases) in men is estimated to be 679,421 in 2020 and 763,575 in 2025. Among women, it is estimated to be 712,758 in 2020 and 806,218 in 2025 (Report of National Cancer Registry Programme, 2020)

Breast cancer in women is estimatedto contribute 200,000 (14.8per cent) and cervix cancer cases about 75,000 (5.4per cent), whereas for both men and women, cancers of the gastrointestinal tract is estimated to contribute 270,000 (19.7per cent) of the total cancer burden.(Global Cancer Statistics, 2020)

Gynecologic cancer is any cancer that starts in a woman's reproductive organs. Cancer is always named for the part of the body where it starts. Gynecologic cancers begin in different places within a woman's pelvis, which is the area below the stomach and in between the hip bones. Gynecologic cancers are malignancies that affect the female organs of reproduction and the genitalia. General risk factors include age, family history, and endogenous or exogenous hormonal factors. These may include age at onset of menstruation, age at or lack of pregnancy, and age at menopause, as well as exposure to hormonal manipulation, including birth control methods and hormonal treatment for gynecologic conditions or symptoms of menopause.. 


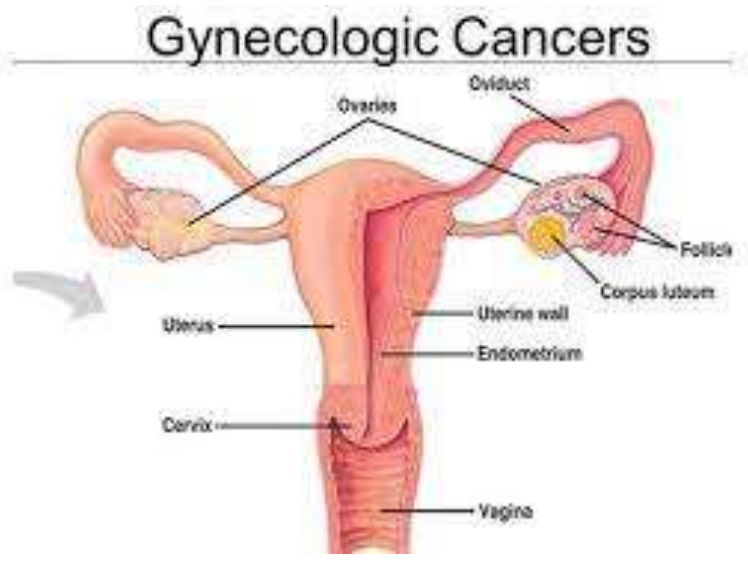

Gynecologic cancer is any cancer that starts in a woman's reproductive organs. While they are often discussed as a group, each gynecologic cancer is unique. The five main types of gynecologic cancer are:

Ovarian cancer-affects the ovaries, a pair of solid, oval-shaped organs producing hormones and eggs (ova).

Uterine cancer-begins in the main body of the uterus, a hollow organ about the size and shape of an upside-down pear. The uterus is where the baby grows when a woman is pregnant.

Cervical cancer-begins in the cervix, the lower, cylinder-shaped part of the uterus. Its upper margin is connected to the uterus, while its lower margin is connected to the vagina.

Vaginal cancer-begins in the vagina (also called the birth canal), a muscular tube-like channel that extends from the cervix to the external part of the females sex organs (vulva).

Vulval cancer-begins in the vulva, the outer part of the female reproductive system. It includes the opening of the vagina, the inner and outer lips (also called labia minora and labia majora), the clitoris and the mons pubis (soft, fatty mound of tissue, above the labia).

Other types of gynecological cancers include fallopian tube cancer and placenta cancer (a pregnancy-related cancer). 


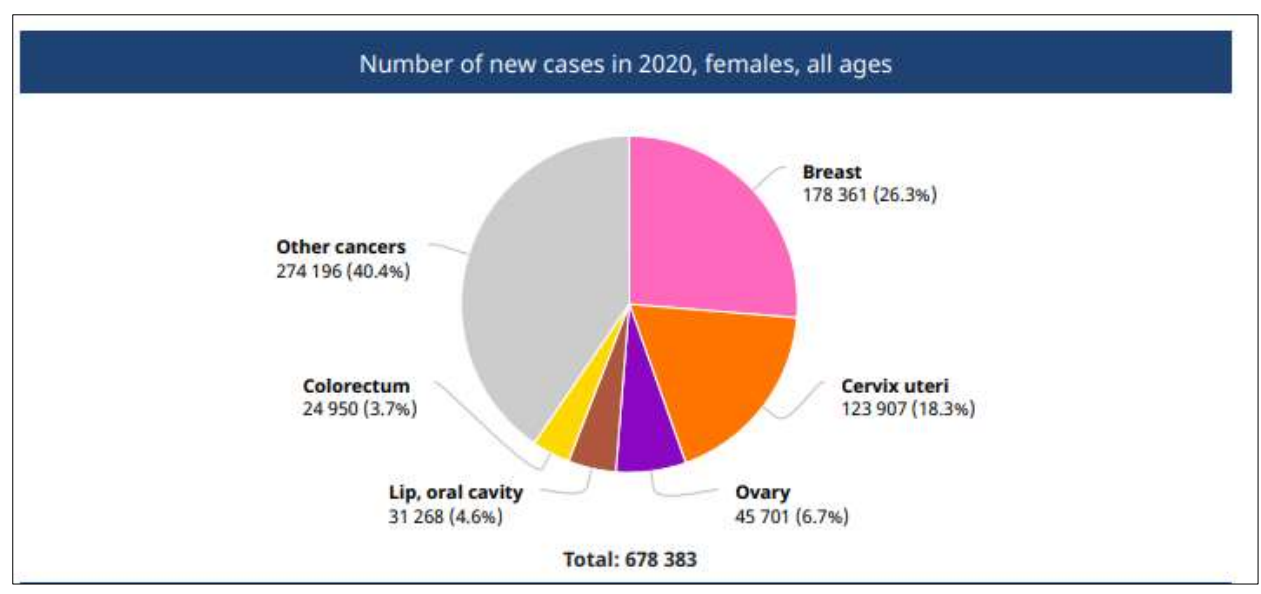

Source:The Global Cancer Observatory ,March, 2021.

\section{Objectives of the study}

1. To understand the Socio-Economic and Demographic Profile of Gynecological Cancer affected Women in Coimbatore District-Tamil Nadu,

2. To analyse the Economic expenditure incurred for Gynecological Cancer treatment in Coimbatore District.

\section{Review of Literature}

Jane Owenga(2018)The number of cervical cancer cases is reported to increase among women of reproductive age in the recent past with patients facing challenges with care and management of the illness. This study assessed financial challenges and sources of financial assistance for cervical cancer patients in western Kenya.Cervical cancer patients experience several financial challenges yet only few of them had insurance cover which catered for only bed component of inpatient costs. There is a need for the Kenyan health care system to develop mechanisms for provision of financial support for cervical cancer patients

Wang (2018) analysed the reproductive system cancer, which cause morbidity and mortality worldwide which threatens women's health and lives.. This study compared and assessed the temporal trends of common female malignances on breast, cervical and ovarian cancer mortality in developed 
regions of Asia including Japan, Republic of Korea and Singapore The mortality risk of three cancers increased with age, and period and cohort effects may collectively affect the common female malignances mortality for East Asian women.

Joohyun Park et al.,(2019)estimated and compared annual health care expendituresper person between non-cancer and cancer patients, and among patients with the four most common cancers. The study found that cancer patients had nearly four times higher mean expenditures per person ( $\$ 16346$ ) than those without cancer (\$4484). These differences were larger among individuals aged 18 to 64 years than those $\geq 65$ years. Medicare was the largest source of payment for cancer patients, especially among those $\geq 65$ years. Among the four most common cancers, the most costly cancer was lung cancer. The study resulted that the cancer patients experienced a substantially higher health care expenditure burden than non-cancer patients, with lung cancer patients having the highest expenditures. Expenditure estimates varied by age group, source of payment, and service type, highlighting the need for comprehensive policies and programs to reduce the costs of cancer care.

Balasubramaniamet al.,(2020)The majority of these cervical patients report at a late-stage of disease in health-care facility due to lack of awareness. A premier tertiary cancer hospital in Mumbai conducted the present survival-study. The study concluded that younger patients, early stage of disease, non-involvement of any sites/ nodes, and radiotherapy either alone or in combination with other treatment type provided better outcomes. Early detection and prevention strategies are keys to obtain better outcomes.

XiaomengYueet al., (2020)the study described the expenditure of gynecologic cancers with treatment patterns and assessed the key risk factors associated with the economic burden in the United States.The estimated annual medical expenditure attributed to gynecologic cancers was $\$ 3.8$ billion, with an average cost of $\$ 6,293$ per patient. The highest annual cost per person was ovarian cancer $(\$ 13,566)$, followed by uterine cancer $(\$ 6,852)$, and cervical 
cancer (\$2,312). The major components of medical costs were hospital inpatient stays (53\%, $\$ 2.03$ billion), followed by office-based visits $(15 \%$, $\$ 559$ million), and outpatient visits (13\%, \$487 million). Two key prescription expenditures were antineoplastic hormones (10.3\%) and analgesics (9.2\%). High expenditures were significantly associated with being a married woman $(p<0.001)$, having private health insurance $(p<0.001)$, being from a low- and middle-income family $(p<0.001)$, or living in the Midwest or the South $(p<0.001)$.

JoesphKazibwe(2021) studied the chronic nature of non communicable diseases (NCD) and costs associated with long-term care can result in the medical expenses on household spendingexceed their actual capacity to bear the expenditure for the patient and the same household pushing them into poverty and widespread inequality in society, the objective of this study was to explore existing evidence on the financial burden of NCDs in low- and middleincome countries (LMICs), specifically estimating the cost incurred by patients with NCDs and their households to inform the development of strategies to protect such households from household spending expenditure.Most of the studies were cross-sectional cost-of-illness studies, of which almost half focused on diabetes and/or conducted in South-East Asia. The average total costs per year to a patient/household in LMICs of COPD, CVD, cancers and diabetes were \$7386.71, \$6055.99, \$3303.81, \$1017.05, respectively. The available evidence on expenditurereveals a large financial deficit imposed on patients and households in seeking and receiving NCD care and emphasizes the need for adequate and reliable social protection interventions to be implemented alongside Universal Health Coverage.

\section{Data Sources and Methodology}

Although studies have been conducted to assess Cancer and its risk factor burden in many regions of India, the data were not compiled together. Understanding this problem, the researcher has planned to conduct the survey 
in Coimbatore city. This city has a multi-cultural society, mostly of the cosmopolitan nature. Its inhabitants are largely conservative and traditional, retaining their roots in their native villages. It is a Municipal Corporation as well as the District Headquarters. The city has numerous hospitals. Apart from the Government hospital, several multi-facility hospitals function in the city. The District Health Department is amongst the best in terms of implementing government-initiated health schemes. Also, several rare surgical procedures have taken place here.

The city also has numerous homeopathic clinics run by NonGovernmental Organizations. The fast pace of industrialization, spiraling population and the increase in the health awareness have led to the growth of the healthcare industry in Coimbatore. In this study, Multi-stage Random Sampling Method was adopted. At the First Stage, in order to fulfill the objectives of the study, the researcher got permission from the Institution's Human Ethics Committee through the study protocol submission form of selected medical institutions with the consent of the respondents. With a view to have a thorough study and understand the Health Status of women respondents, Government Hospital and four private hospitals in Coimbatore City were selected. A pilot study was conducted with 76 women cancer patients.

At the Second Stage, in order to select representative patients among women population receiving treatment in these hospitals, it was felt an empirical inquiry would be apt. The Patients whohad visited the Five Hospitals have been taken as sample respondents. From that list who were registered as cancer patients in general and in particular as women. The total number of the Gynecological women cancer patients had been identified and those who have undergone the treatment of Surgery, Chemotherapy, Radiation and Taking Tablets were listed out. It was identified that 6,540 women cancer patients had treatment seeking behavior and are approaching the physician regularly. It was found that Gynecological Related Cancer were collectively named as Gynecological Cancer Patients $(2,090)$.The total number of cancer patients in 
types of cancer was 6,540. For a meaningful selection, 10 per cent of the women cancer patients were selected randomly from four Private Hospitals and one Government hospital in Coimbatore District.

At the Third Stage, an Interview schedule - prepared for the purpose of data collection consisted of questions relating to socio - economic and demographic profile, treatment cost incurred for cancer treatment in prehospitalization, hospitalization and post hospitalization period. Total Cost incurred for treatment is analysed through Multiple Linear Regression.

\section{Results and Discussions}

Total Cost of Cancer Treatment-Multiple Regression Analysis

Multiple Regression Analysis refers to a set of techniques for studying the straight-line relationships among two or more variables. Multiple regression estimates the $\beta^{\prime} s$ in the equation

$$
y j=0+1 \times 1 j+-2 \times 2 j+\ldots+p x p j+j
$$

The $X^{\prime}$ 's are the independent variables (IV's). $Y$ is the dependentvariable. The subscript $j$ represents the observation (row) number. The $\beta$ 's are the unknown regressioncoefficients. Their estimates are represented by $b$ 's. Each $\beta$ represents the original unknown (population) parameter, while $b$ is an estimate of this $\beta$. The $\varepsilon j$ is the error (residual) of observation $j$. Although the regression problem may be solved by a number of techniques, the most-used method is least squares. In least squares regression analysis, the $b^{\prime}$ sare selected so as to minimize the sum of the squared residuals. This set of $b^{\prime}$ sis not necessarily the set you want, since they may be distorted by outliers--points that are not representative of the data. Robust regression, an alternative to least squares, seeks to reduce the influence of outliers.

Multiple regression analysis studies the relationship between a dependent (response) variable and $\mathrm{p}$ independent variables (predictors, regressors, IV's). The sample multiple regression equation is 


$$
\hat{y} \quad j \cdot b 0+b 1 \times 1 j+b 2 \times 2 j+\ldots+b p \times p j
$$

If $p=1$, the model is called simple linear regression.

The intercept, $b 0$, is the point at which the regression plane intersects the

The $b i$ are the slopes of the regression plane in the direction of $x i$. These coefficients are called the partial-regression coefficients. Each partial regression coefficient represents the net effect the $i^{\text {th }}$ variable has on the dependent variable, holding the remaining $X^{\prime}$ s in the equation constant.

A large part of a regression analysis consists of analyzing the sample residuals, ej, defined as $e j=y j-y j$. Once the $\beta$ 's have been estimated, various indices are studied to determine the reliability of these estimates. One of the most popular of these reliability indices is the correlation coefficient. The correlation coefficient, or simply the correlation, is an index that ranges from 1 to 1 . When the value is near zero, there is no linear relationship. As the correlation gets closer to plus or minus one, the relationship is stronger. A value of one (or negative one) indicates a perfect linear relationship between two variables.

The regression equation is only capable of measuring linear or straight-line relationships.

If the data form a circle, for example, regression analysis would not detect a relationship. For this reason, it is always advisable to plot each independent variable with the dependent variable, watching for curves, outlying points, changes in the amount of variability, and various other anomalies that may occur. If the data are a random sample from a larger population and the $\varepsilon j$ are independent and normally distributed, a set of statistical tests may be applied to the $b$ 'sand the correlation coefficient. These $t$-tests and $F$-tests are valid only if the above assumptions are met. The F-ratio, which is computed from the mean squared terms in the Analysis of variance (ANOVA) table, estimates the statistical significance of the regression equation. 


$$
\mathrm{F}=\mathrm{MSR} / \mathrm{MSE}
$$

where MSR mean square error of regression and MSE mean square error of the residuals.

Table 1

Total Treatment Costs of All Cancers - Multiple Linear Regression Analysis

\begin{tabular}{|l|c|}
\hline \multicolumn{1}{|c|}{$\begin{array}{c}\text { Explanatory } \\
\text { Variables }\end{array}$} & $\begin{array}{c}\text { Standardiz } \\
\text { ed Regression } \\
\text { Coefficients (B) }\end{array}$ \\
\cline { 2 - 3 } & $\begin{array}{c}\text { Gynecological } \\
\text { Cancer }\end{array}$ \\
\hline Age (in Years) & $-0.421^{* * *}$ \\
\hline Family Size (in Members) & -0.077 \\
\hline $\begin{array}{l}\text { Educational Qualification } \\
\text { (in 6 levels) }\end{array}$ & $0.187^{* * *}$ \\
\hline $\begin{array}{l}\text { Monthly Personal Income } \\
\text { (in Rs.) }\end{array}$ & $0.256^{* * *}$ \\
\hline Nativity (Ref: Rural)Urban & $-0.179^{* * *}$ \\
\hline $\begin{array}{l}\text { Religion (Ref: } \\
\text { Others)Christians }\end{array}$ & $0.288^{* * *}$ \\
\hline $\begin{array}{l}\text { Social Class (Ref. SC/ST) } \\
\text { Others Castes }\end{array}$ & -0.048 \\
\hline $\begin{array}{l}\text { Type of Cancer (Ref: } \\
\text { Others) } \\
\text { Breast Cancer }\end{array}$ & -- \\
\hline Type of Cancer (Ref: & \\
\hline
\end{tabular}




\begin{tabular}{|c|c|}
\hline $\begin{array}{l}\text { Others) } \\
\text { Gynecological Cancer }\end{array}$ & \\
\hline R2 (in ) & $\mathbf{4 4 . 6}$ \\
Total Sample & $\mathbf{2 0 9}$ \\
\hline
\end{tabular}

Note: -- = Not included in the Models.

$+,{ }^{*},{ }^{*}$ and ${ }^{* * *}=\mathrm{t}$-statistics related to the concerned Beta Coefficients are

Significant at $0.10,0.05,0.01$ and 0.001 levels, respectively.

For this purpose, the total cost incurred for treatment considered here as dependent variable, which is measured as discrete or continuous in nature (i.e., actual total cost incurred Indian Rupees-sum of direct and indirect costs). The independent (explanatory) variables thought out here for analysis are selected based on the theoretical importance as well as their levels of significance with the dependent variable. Of the nine variables included in the model, four are continuous (or discrete) in nature and the other five are dummy variable type (two categories only - for details see Table1).

Information about the amount spent for gynecological cancer by women patients is given in of Table 1. From this, one can observe that all the seven variables under consideration together have explained 44.6 percent of variation in the total cost incurred for the treatment of gynecological cancer by sample women patients. Controlling for all the variables included in the model, the cost for treatment of the gynecological cancer is noted to be on the increasing side with an increase in their monthly personal income $(\beta=0.256 ; p<0.001)$ and level of education $(\beta=0.187 ; p<0.001)$. Next to these, as expected the cost spent for gynecological cancer observed as profoundly higher among those belonged to Christianity $(\beta=0.288 ; p<0.01)$ compared to those who belonged to Hinduism and Muslim.

Among the other factors under consideration, as noted earlier, age has exhibited a striking negative net effect on the cost incurred for gynecological cancer $(\beta=-0.421 ; p<0.001)$, i.e., there is clear pattern of decreasing cost for 
gynecological cancer with an increase in the current age of women patients. Another prominent finding noted here is that the cost of treatment for gynecological cancer is observed to be much less among those residing in urban areas $(\beta=-0.179 ; p<0.001)$ than those who are dwelling in rural areas. On the other hand, contrary to the expectation, on the one side the cost incurred for the gynecological cancer is relatively on the increase side with an increase in the family size of the sample women under consideration, whereas on the other side such amount is comparatively lower among those who belonged to non-SC / ST communities as against to those who belonged to SC / ST communities; but the t-test results in both these case didn't turn out as statistically significant. The results obtained in this study are Devi (2009), Peter, (2009).

\section{Conclusion}

Gaps remain in the understanding of why Gynecological Cancer patients have higher expenditures than are seen with other common tumours. Gynecological Cancer patients are predominately female, and yet, in the regression analysis, sex was not a good predictor of Out of Pocket Cost. The researcher has noted that insurance coverage is a factor: it remained in the final regression model for this analysis as it did in earlier publications. The researcher noted that people under the age of 65 are less likely to have comprehensive coverage because many health programs are not covered through the public purse until a person reaches the age of 65 , and this factor is likely plays an important role. The results obtained in this study are the same as byZarogoulidou (2015) and Ward(2003).As expected the cost incurred for the other cancers is comparative on the increase side with an increase in the family size of the sample women, but the t-test results in this regard found to be insignificant. 


\section{References}

1. AswathySreedevi\&ReshmaJavedandAvani Dinesh.(2015). "Epidemiology of cervical cancer with special focus on India", International Journal of Women's Health 405-414.

\section{Balasubramaniam G, Gaidhani RH, Khan A, Saoba S, Mahantshetty}

U, Maheshwari A.(2020)"Survival rate of cervical cancer from a study conducted in India" Indian Journal of Medical Sciences, doi: 10.25259/JJMS_140_2020.

3. C S Pramesh (2014) "Cancer burden and Health systems in India 3 Delivery of affordable and equitable cancer care in India" DOI:10.1016/S1470-2045(14)70117-2

4. Habtu et al. (2018) "Health seeking behavior and its determinants for cervical cancer among women of childbearing age in Hossana Town, Hadiya zone, Southern Ethiopia: community based cross sectional study" BMC Cancer, 18, 298

5. Jane AOwenga\& Erick OtienoNyambedha(2018), "Perception of Cervical Cancer Patients on their Financial Challenges in Western Kenya" BMC Health Service Research published in 10 April 2018.

6. JoesphKazibwe (2021) "The Household financial burden of noncommunicable diseases in low -and middle -income countries : a systematic review PMID: 34154609 PMCID: PMC8215836 DOI: 10.1186/s12961-021-00732-y

https://pubmed.ncbi.nlm.nih.gov/34154609/

7. Joohyun Park \& Kevin (2019) "Health Care Expenditure Burden of Cancer Care in the United States" The Journal of Health Care Organization, Provision, and Financing, 56, 1-9.

8. Kaur, H. \&Bisht, B. (2014) "Assessing the Impact of Awareness program on Breast and Cervical Cancer Knowledge Empowerment among Working Women in Education Sector" Journal of Multidisciplinary Research in Healthcare, 1(1), 19-31. 
9. Maheshwari A, Kumar N, Mahantshetty U. (2016), "Gynecological cancers: A summary of published Indian data" South Asian Journal Cancer;5:112-20.

10. Sharma , Das et.al (2009) "Economic cost analysis in cancer management and its relevance Today" Review Article, Volume 46, Issue 3 PP-184-189

11. Will, B. P., Berthelot, J. M., Nobrega, K. M., Flanagan, W., \& Evans, W. K. (2001) "Canada's Population Health Model (POHEM): A tool for performing economic evaluations of cancer control interventions" European Journal of Cancer,37(14), 1797-1804.

\section{XiaonmengYue, Jane $M$ Pruemer, Ana I Hincape, Ziyad S} AlmalikJefv J Guo(2020)"Economic burden and treatment patterns of gynecologic cancers in the United States: evidence from the Medical Expenditure Panel Survey 2007-2014", Jul;31(4):e52. doi: 10.3802/jgo.2020.31.e52. Epub 2020 Mar 6. 